\title{
SCHRÖDINGER EQUATION FOR A DIRAC BUBBLE POTENTIAL
}

\author{
S.M. BLINDER \\ Department of Chemistry, Lniversiry of Michigan, Ann Arbor, Michigan 48109, USA
}

Received 19 February 1979

\begin{abstract}
The quantum-mechanical problem of a particle moving in a "Dirac bubble potential" $U(r)=\left(\lambda / r_{0}\right) \delta\left(r-r_{0}\right)$ is solved exactly for both bound and continuum states by making use of partial wave Green's functions $G_{l}\left(r . r_{0} k\right)$. Phase shifts are expressed in a compact form related to those for an impenetrable sphere.
\end{abstract}

Recently I worked out a closed-form solution to the Schrödinger equation for a inydrogen atom perturbed by a modification of the Fermi contact interaction [1]. Specifically, the perturbation added to the Coulomb potential is

$\mathscr{H}^{\prime}=\alpha \delta\left(r-r_{0}\right) / 4 \pi r_{0}^{2}, \quad \alpha \equiv \frac{8}{3} \pi \mu_{S} \cdot \mu_{I}$,

in which $\mu_{S}$ and $\mu_{I}$ are, respectively, electron spin and nuclear spin magnetic moment operators. The nuclear moment in this model is idealized as a magnetic shell (or bubble) of radius $r_{0}$. As $r_{0}$ is decreased to zero, the nucleus approaches a point magnetic dipole and eq. (1) reduces to the conventional Fermi contact interaction operator $\mathscr{H}^{\prime \prime}=\alpha \delta^{3}(r)$.

We shall consider in this paper a simpler version of the problem without the Coulomb potential. The results will add another to the small number of exactly soluble quantum-mechanical problems. The Schrödinger equation for a particle in a Dirac bubble potential is written:

$\left[-\frac{\hbar^{2}}{2 m} \nabla^{2}+\frac{\alpha}{4 \pi r_{0}^{2}} \delta\left(r-r_{0}\right)\right] \psi(r)=E \psi(r)$

Spherical symmetry allows the factorization $\psi(r)=$ $R_{k l}(r) Y_{l m}(\theta, \phi)$. The radial equation can be arranged to

$$
\begin{aligned}
& {\left[k^{2}+\frac{1}{r^{2}} \frac{\partial}{\partial r} r^{2} \frac{\partial}{\partial r}--\frac{l(l+1)}{r^{2}}\right] R_{k l}(r)} \\
& =\left(\lambda / r_{0}\right) R_{k l}\left(r_{0}\right) \delta\left(r-r_{0}\right),
\end{aligned}
$$

having defined

$E \equiv \hbar^{2} \dot{\kappa}^{2} / 2 m, \quad \lambda \equiv m \alpha / 2 \pi \hbar^{2} r_{0}$.

We observe that eq. (3) is isomorphous with the defining relation for the lth partial wave Green's function

$$
\left[k^{2}+\frac{1}{r^{2}} \frac{\partial}{\partial r} r^{2} \frac{\partial}{\partial r}-\frac{l(l+1)}{r^{2}}\right] G_{l}\left(r, r_{0}, k\right)=\delta\left(r-r_{0}\right) / r_{0}^{2}
$$

In standard Sturm-Liouville form:

$$
\left[\frac{\partial}{\partial z} z^{2} \frac{\partial}{\partial z}+z^{2}-l(l+1)\right] G_{l}=k \delta\left(z-z_{0}\right), \quad z \equiv k r .
$$

The solution to (6) regular at $z=0$ with outgoing wave behavior as $z \rightarrow \infty$ is a product of spherical Bessel functions:

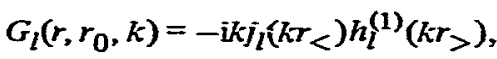

in which $r_{>}$and $r_{<}$are, respectiveiy, the larger and smaller of $r, r_{0}$.

Outgoing wave solutions to the Schrödinger equation (3) with $E>0$ can accordingly be represented in the form

$$
R_{k l}(r)=A j_{l}(k r)-i \lambda k r_{0} R_{k l}\left(r_{0}\right) j_{l}\left(k r_{<}\right) h_{l}^{(1)}\left(k r_{>}\right) .
$$

The first term (complementary function) is a solution of the free-particle equation regular at $r=0$. For consistency at $r=r_{0}$, the constant $A$ must fulfil the condition 


$$
A j_{l}\left(k r_{0}\right)=R_{k l}\left(r_{0}\right)\left[1+i \lambda k r_{0} j_{l}\left(k r_{0}\right) h_{l}^{(1)}\left(k r_{0}\right)\right] \text {. }
$$

The magnitude of $R_{k l}\left(r_{0}\right)$ determines the normalization of the wavefunction.

For $r>r_{0}$, the eigenfunc \pm ions (8) can be expressed in terms of phase shifts $\delta_{l}$ as follows:

$R_{k l}(r)=\operatorname{const}\left[j_{l}(k r) \cos \delta_{l}-n_{l}(k r) \sin \delta_{l}\right]$.

With use of (9) it is shown that

$\operatorname{ctn} \delta_{l}=\frac{n_{l}\left(k r_{0}\right)}{i_{l}\left(k r_{0}\right)}-\frac{1}{\lambda k r_{0}\left[i_{l}\left(k r_{0}\right)\right]^{2}}$.

As $\lambda \rightarrow \infty$, the bubble becomes opaque and (11) reduces to the well-known result for scattering by an impenetrable sphere [2.p. 38]:

$\operatorname{ctn} \bar{\xi}_{l}=n_{l}\left(k r_{0}\right) / \bar{l}_{l}\left(k r_{0}\right)$.

In the limit $\lambda \rightarrow 0$, on the other hand, one obtains the Born approximation phase shifts $[2$, p. 89]

$\eta_{l}=-\frac{2 m k}{\hbar^{2}} \int_{0}^{\infty} V(r)\left[j_{l}(k r)\right]^{2} r^{2} d r=-\lambda k r_{0}\left[j_{l}\left(k r_{0}\right)\right]^{2}$.

Putting (12) and (13) into (11), one can express the phase shifts $\delta_{2}$ in the compact form

$\operatorname{ctn} \delta_{l}=\operatorname{ctn} \xi_{l}+n_{l}^{-1}$.

For consideration of the $E<0$ bourd states of a bubble potential, it is convenient to introduce the variable

$\kappa \equiv-i k, \quad E=-\hbar^{2} k^{2} / 2 m$.

The Green's function ( 7 ) can be written

$G_{l}\left(r_{2} r_{0}, k\right)=-k S_{l}\left(k r_{<}\right) X_{l}\left(k r_{>}\right)$

in terms of modified spherical Bessel functions de-

fined as follows:

$\mathscr{S}_{1}(z) \equiv(\pi / 2 z)^{1 / 2} I_{I+1 / 2}(z)=\hat{i}^{-l} j_{l}(i z)$,

$x_{l}(=) \equiv(2 / \pi z)^{1 / 2} K_{l+1 / 2}(z)=-i^{I} h l^{(1)}(\bar{i} z)$.

Specifically, the first three functions of each type are [3]

$9_{0}(z)=z^{-1} \sinh z$

$S_{1}(z)=z^{-1} \cosh z-z^{-2} \sinh z$, $\vartheta_{2}(z)=\left(z^{-1}+3 z^{-3}\right) \sinh z-3 z^{-2} \cosh z$

and

$$
\begin{aligned}
& X_{0}(z)=z^{-1} \mathrm{e}^{-z} \\
& \chi_{1}(z)=\left(z^{-1}+z^{-2}\right) \mathrm{e}^{-z} \\
& X_{3}(z)=\left(z^{-1}+3 z^{-2}+3 z^{-3}\right) \mathrm{e}^{-z} .
\end{aligned}
$$

For bound states the Schrödinger equation (3) has the solutions

$R_{k l}(r)=-\lambda k r_{0} R_{k l}\left(r_{0}\right) \mathscr{I}_{l}\left(k r_{<}\right) \mathcal{X}_{l}\left(k r_{>}\right)$,

there being no complementary function for $E<0$. For $r=r_{0}$, the consistency condition on eq. (20),

$-\lambda k r_{0} \rho_{l}\left(\kappa r_{0}\right) X_{l}\left(k r_{0}\right)=1$

provides a transcendental equation determining the eigenvalues of the problem.

For $z \geqslant 0$, both $\mathcal{S}_{l}(z)$ and $\mathcal{X}_{l}(z)$ are positive definite. Thus (21) has real solutions corresponding to bound states only when $\lambda<0$. This is physically reasonable since the latter condition implies an attractive potential. Eq. (21) can be cast in the form

$z \mathcal{O}_{l}(z) \mathcal{K}_{l}(z)=|\lambda|^{-1}, \quad=z \equiv \kappa r_{0}$.

From formulas given in ref. [3], the left-hand side is verified to be a monotonically-decreasing function of $z$ (for $z \geqslant 0$ ) with a maximum value $(2 l+1)^{-1}$ at $z=0$. As $z \rightarrow \infty . z g_{1}(z) X_{1}(z) \sim 1 / 2 z$.

It follows that there exists one bound state for each angular momentum, so long as

$|\lambda|>2 l+1$.

For $|\lambda|<2 l+1$, eq. (22) has no solution with $z>0$, hence no bound states will exist above some critical value of $l$.

For $l=0$, eq. (21) reduces to the simple form

$\left(1-e^{-2 k r_{0}}\right) / 2 k r_{0}=|\lambda|^{-1}$

\section{References}

[1] S.M. Blinder, Phys. Rey. A18 (1978) 853.

[2] N.F. Mott and H.S.W. Massey, The theory of atomic collisions, 3rd Ed. (Oxford Univ. Press, London, 1965).

[3] M. Abramowitz and I.A. Stegun, eds., Handbook of mathematical functions (NatI. Bur. Std., Washington, 1972) p. 443. 